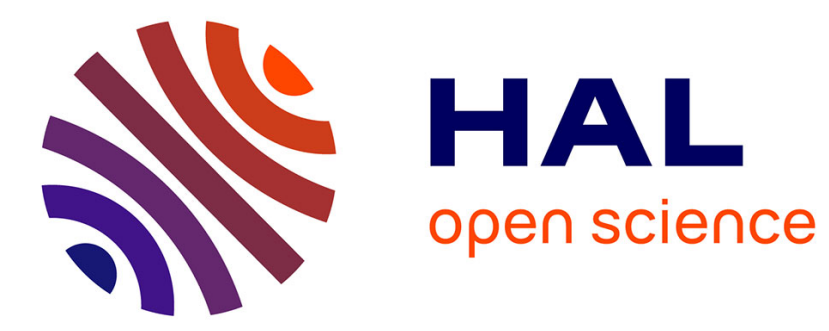

\title{
Whistler Emission by Electron Beams
}

C. Krafft, B. Lundin, G. Matthieussent, A. Volokitin

\section{To cite this version:}

C. Krafft, B. Lundin, G. Matthieussent, A. Volokitin. Whistler Emission by Electron Beams. Journal de Physique IV Proceedings, 1995, 05 (C6), pp.C6-37-C6-42. 10.1051/jp4:1995607 . jpa-00253970

\section{HAL Id: jpa-00253970 https://hal.science/jpa-00253970}

Submitted on 1 Jan 1995

HAL is a multi-disciplinary open access archive for the deposit and dissemination of scientific research documents, whether they are published or not. The documents may come from teaching and research institutions in France or abroad, or from public or private research centers.
L'archive ouverte pluridisciplinaire HAL, est destinée au dépôt et à la diffusion de documents scientifiques de niveau recherche, publiés ou non, émanant des établissements d'enseignement et de recherche français ou étrangers, des laboratoires publics ou privés. 


\title{
Whistler Emission by Electron Beams
}

\author{
C. Krafft, B. Lundin*, G. Matthieussent and A. Volokitin* \\ Laboratoire de Physique des Gaz et des Plasmas, Bât. 212, Université Paris-Sud, CNRS, \\ 91405 Orsay Cedex, France \\ * Institute of Terrestrial Magnetism, Ionosphere and Radiowave Propagation, Academy \\ of Sciences, Troitsk, Moscow Region, 142092, Russia
}

\begin{abstract}
Experimental and theoretical investigation of whistler waves produced in the interaction of a density modulated electron beam with a magnetized plasma are presented for the conditions of active space experiments in the Earth ionosphere as well as for the case of overdense laboratory plasmas. Structures of the electromagnetic fields far and near the beam, efficiency of the beam modulation, emitted power are examined as well as conditions for an undistorted registration of the whistler signals by remote receivers in space.
\end{abstract}

\section{INTRODUCTION}

The interactions of electron beams with plasmas are traditional subjects of both laboratory and space research. For active experiments in the near Earth plasma dealing with modification of the radiation belts or aimed at communication purposes, one of the most attractive frequency range is the electron whistler frequency band. The waves of this mode can propagate over a long distance in the magnetosphere being guided along the Earth magnetic field lines. They play an important role in establishing a natural equilibrium state in the Earth radiation belts [1]. However, most active experiments dealing with the influence of whistler waves on the Earth radiation belts [2] are limited by the inefficiency of exciting these waves even through the use of a large antenna on the Earth's surface. This is due to their very long wavelengths and strong absorption in the lower ionosphere.

The emission of whistler waves directly in space with the help of such large-scale structures as energetic particle beams could increase manifold the amplitudes of the wave field in the ionosphere as well as in the distant magnetosphere. Since the ARAKS experiment showed that the wave emissions came mainly from the beam front [3], an artificial modulation of the beam density at some appropriate frequency should also increase the efficiency of beams as extended antennas in space for radiation of very long wavelength signals. Experiments with modulated electron beams artificially injected into magnetized plasmas were performed recently in a laboratory [4-7] and in space during the Active Plasma EXperiment (APEX) program [8].

Theoretical and experimental approaches have been used to investigate the efficiency of the beam modulation to excite whistler emission in a dense ionospheric or laboratory plasma. New experimental results were obtained about dispersive properties and spatial distribution of the whistler wave field excited by a modulated electron beam in an afterglow bounded laboratory plasma [4-7]. In the far field approximation, peculiarities of the space-time structure of the emitted field have been described during and after the beam front pass; the problem of efficiency of the nonmonoenergetic beam emission has been studied [9-11]. The analysis of the electromagnetic field in the near and far regions from the electron beam has also been performed, and the power of emission could be estimated [12-14].

\section{LABORATORY EXPERIMENT [4-7]}

The experiment (see set-up on Fig. 1a) is performed in an Argon discharge plasma generated by a large negatively biased oxyde cathode which provides electrons accelerated by an adjacent grounded grid, 
which ionize the gas. The plasma column ( $70 \mathrm{~cm}$ length, $30 \mathrm{~cm}$ radius) is immersed in a homogeneous axial magnetic field less than $300 \mathrm{G}$. Pulsed discharges are used [5] and the experiment is performed in the afterglow which provides a Maxwellian plasma with the following characteristics: $10^{10} \leq n_{p} \leq 10^{12} \mathrm{e} / \mathrm{cm}^{3}$ (plasma density), $0.2 \leq T_{e} \leq 2 \mathrm{eV}$ (electron temperature), $f_{R}=5 \mathrm{~Hz}$ (repetition rate of discharge), $P=10^{-4} \mathrm{~T}$ (Argon pressure). The electron gun, which is a commercially available triode, is located in a separate chamber at the end of the main chamber opposite to the cathode. It can deliver a narrow electron beam which is injected in the afterglow plasma (see Fig. 1b) with typical parameters: $n_{b} \approx 10^{8} \mathrm{e} / \mathrm{cm}^{3}$ (beam density), $I_{b} \leq 10 \mathrm{~mA}$ (beam current), $E_{b} \leq 300 \mathrm{eV}$ (beam energy), $r_{b}=3.5 \mathrm{~mm}$ (beam radius), $\omega_{0} \approx 100 \mathrm{MHz}$ (modulation frequency), $\tau_{b}=10 \mu \mathrm{s}$ (pulse duration), $\alpha \leq 15^{\circ}$ (pitch angle). Movable Langmuir probes and shielded magnetic loops are used to measure the electric and magnetic wave fields.
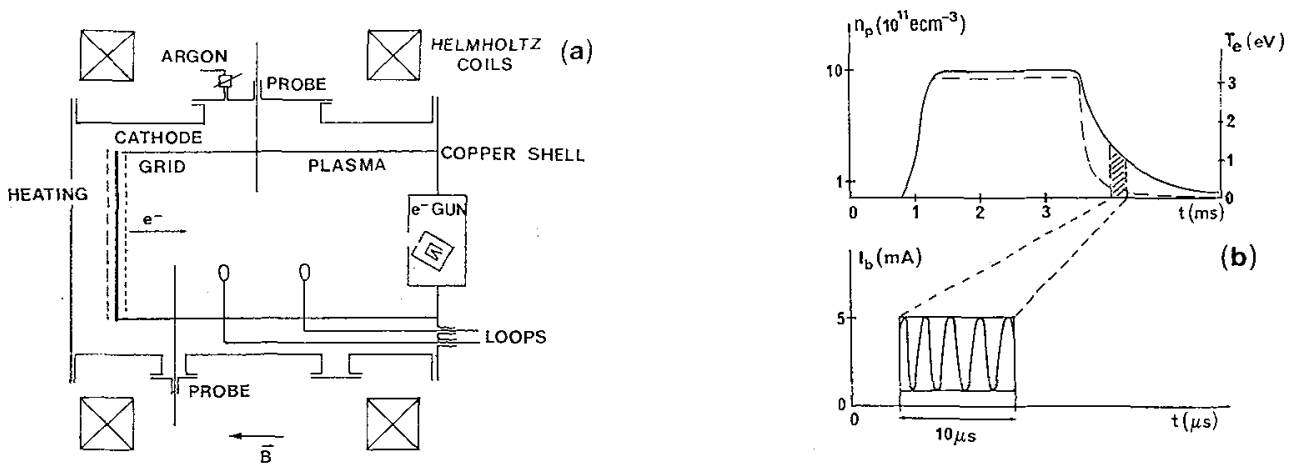

Figure 1: (a) Schematic representation of the experimental set-up; (b) Typical temporal evolution of the plasma density (solid line) and temperature (dashed line) showing the modulated beam injection.

Whistler waves can be excited in Cherenkov resonance by a density modulated beam [6-7] with a maximum of signal on the double pole (beam mode and plasma mode together, see also Fig. 2a) compared to the excitation at a single pole (plasma mode). Parametric studies of the wave emission have been performed and compared to excitation of whistlers by magnetic loops. Electric and magnetic field profiles were measured and compared with a numerical study of fields excited in a bounded plasma. We have shown that the whistlers propagate quasi-parallel to the magnetic field by measurements of the phase planes. Beam modes could be evidenced when the modulation frequency was greater than the electron gyrofrequency $\omega_{c}$. We have exhibited a beat between the plasma mode and the beam mode at high plasma densities: this is in agreement with the theoretical model developed for the experiment (see below). The field amplitude was estimated through magnetic loop calibration: $1 \mathrm{mV}$ on the loop corresponds to an electric field of $810^{2} \mathrm{Vm}^{-1}$. In conclusion, the linear stage of the interaction has been studied and an increase of the modulation current should allow to reach the nonlinear regime in order to study the saturation mechanisms of the emission.

Let us present briefly the theoretical model which explains the experimental results [7]. We can use a one dimensional model to describe the waves and the beam in the bounded plasma, since phase planes are perpendicular to the magnetic field directed along the axis $O_{z}$; so all radial dependance can be neglected and the modulated beam current can be expressed as $j(z, t)$, which leads to an electric field $E(z, t)$

$$
j(z, t)=j_{0} \sin \left[\omega_{0}(t-z / v)\right] Y(t-z / v) Y(z), \quad E(z, t)=\int \frac{e^{-i(\omega t-k z)} d \omega d k}{\omega\left(\omega-\omega_{0}\right)(k-\omega / v) \varepsilon(\omega, k)}
$$

where $v$ is the beam velocity. We can distinguish in this integral as well as on the dispersion curves (see Fig. 2a), the poles corresponding to the modulation frequency, to the Cherenkov resonance of the beam and to the plasma dispersion relation (dielectric constant $\varepsilon(\omega, k)$ ). 
Two cases can occur (see Fig. 2a): (i) if the beam velocity is equal to $v_{1}$, the waves are excited at point $A$ as whistler modes and at point $M$ as beam modes: two single poles exist and we can calculate the ratio $\left|E_{A}\right| /\left|E_{M}\right|$ of the corresponding electric fields. Figure $2 \mathrm{~b}$ shows its variation as a function of the ratio $\nu / v_{\varphi}$ of the beam velocity to the whistler phase velocity: first, when the phase velocity is small, (at high plasma densities), a beat appears between the beam and the plasma modes; second, for lower ratios of velocities, the plasma mode is excited preferentially. These two points were evidenced in the experiment. (ii) If the beam velocity is equal to $v_{2}$, then the point A excited represents a whistler wave with phase velocity equal to the beam velocity. The amplitude of the electric field at A grows linearly with $z$.
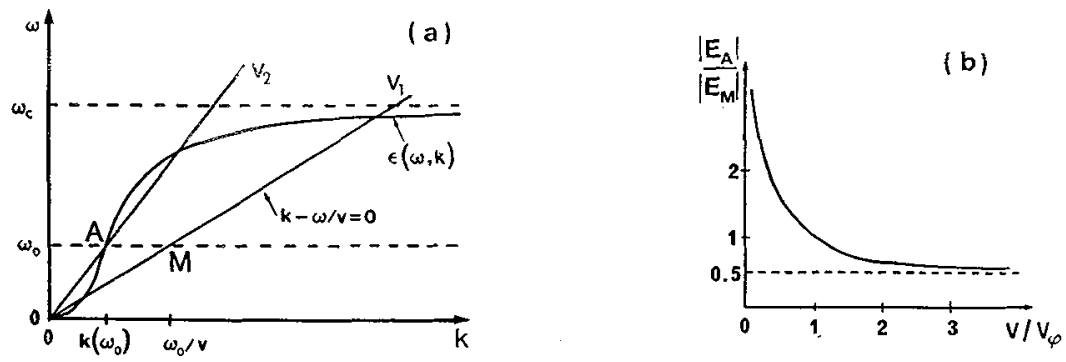

Figure 2: (a) Dispersion curves of the beam-whistler system; (b) Calculated variation of $\left|E_{A}\right| /\left|E_{M}\right|$ with $v / v_{\varphi}$.

\section{WHISTLER EMISSION IN SPACE [9-11]}

Parallel to the laboratory experiment, a theoretical study on the whistlers produced by a thin modulated electron beam injected in the ionospheric plasma has been performed in order to apply the results to active space experiments. In the APEX experiment [8], two satellites were launched by russian physicists: the main satellite contains the gun which injects the electron beam; the subsatellite receives the whistler signals at position $(R, t)$, with distances $R$ and $Z$ to the main satellite respectively accross and along the external magnetic field.

In the cold approximation for a dense plasma $\left(\omega_{p}>\omega_{c}, \omega_{p}\right.$ is the plasma frequency), and for a semiinfinite thin modulated electron beam of current $I\left(t_{0}\right)=I_{0}\left(1-\chi \exp \left(-v t_{0}\right) \cos \left(\omega_{0} t_{0}\right)\right)$ injected at time $t_{0}$ with the modulation frequency $\omega_{0}$ and the modulation amplitude $\chi$ ( $v$ is a small damping factor), one obtains the radiated field as

$$
\begin{gathered}
E=\frac{I_{0}}{e} \sum_{m=-\infty}^{\infty} \sum_{j=1}^{2} \int_{-\infty}^{\infty} d \omega \exp [i \tilde{F}(\omega, u)] \tilde{G}_{m}(\omega)\left[\frac{i}{\omega}-\frac{\chi}{2}\left(\frac{i}{\left(\omega-\omega_{0}+i v\right)}+\frac{i}{\left(\omega+\omega_{0}+i v\right)}\right)\right] \\
\tilde{F}(\omega, u)=-\omega(t-Z / u)+(-1)^{j+1} k_{\perp j}(\omega) R+m Z \omega_{c} / u \\
\tilde{G}_{m}(\omega)=G_{m}(\omega) \exp \left[i(-1)^{j+1} k_{\perp j}(\omega) R\right]
\end{gathered}
$$

where $u$ is the parallel beam velocity and $e$ the electron charge. The field appears as a summation over all resonances $m\left(k_{z} u=\omega+m \omega_{c}\right)$ and whistler branches $j\left(k_{\perp j}\right.$ are the solutions of the dispersion equation) of an integral over the frequency $\omega$. We use the Stationary Phase Point (SPP) method to calculate this integral, in the case of far field approximation $\left(k_{\perp j} R>1\right)$. The main contribution to this integral results from the residue near the modulation frequency and from the integration in the vicinity of the SPP frequencies $\omega_{S}$ defined as solutions of $\partial \tilde{F}(\omega, u) / \partial \omega=0$. The roots of this equation determine the frequencies of the received signal as a function of time and mutual position of gun and receiver. It corresponds to the approximation where the main bulk of the emitted field propagates with the group velocity $\vec{v}_{G}\left(v_{G Z}, v_{G L}\right)$ from the beam front to the receiver. When the modulation and the SPP frequencies are sufficiently far one from the other, the formula for separate contribution of the residues at $\omega_{0}$ and SPP 
can be used. In a general case, we calculate the contribution of the SPP near the pole by modifying the usual SPP method to avoid the singularity near the pole of integrand. The electric field is then obtained as [10]

$$
\begin{gathered}
E=\frac{I_{0}}{e}\left(-i \frac{\chi}{2} \mathrm{I}_{1}+\mathrm{I}_{2}\right)+c . c . \\
\mathrm{I}_{1}=\sum_{m=-\infty}^{\infty} \sum_{j=1}^{2} \exp \left[i F\left(f_{S}\right)\right]\left(G_{m}\left(\omega_{0}\right) i \pi w(y)+\sqrt{\pi} \delta f \exp \left(i F_{2}\right)\left(\frac{G_{m}\left(\tilde{\omega}_{0}\right)}{\left(f_{0}-f_{S}\right) F_{3}}-\frac{G_{m}\left(\omega_{S}\right)}{f_{0}-f_{S}}\right)[1+O(1 / R)]\right) \\
\mathrm{I}_{2}=\sum_{m=-\infty}^{\infty} \sum_{j=1}^{2} \exp \left[i F\left(f_{S}\right)\right] \sqrt{\pi} \delta f \exp \left(i F_{2}\right) G_{m}\left(\omega_{S}\right)\left[\frac{i}{f_{S}}-\frac{\chi}{2} \frac{i}{\left(f_{S}+f_{0}^{*}\right)}\right] \\
y=\frac{\left(f_{0}-f_{S}\right)}{\delta f} F_{3} \exp \left(-i F_{2}\right), \quad w(y)=\frac{i}{\pi} \int_{-\infty}^{\infty} \frac{e^{-y^{2}}}{y-t} d t, \quad \delta f=\sqrt{\frac{2}{\left|F^{\prime \prime}\left(f_{S}\right)\right|}} \\
F_{2}=\frac{\pi}{4} \operatorname{sign}\left[F^{\prime \prime}\left(f_{S}\right)\right], \quad F_{3}=1+\left(f_{0}-f_{S}\right) F^{\prime \prime \prime}\left(f_{S}\right) / 6 F^{\prime \prime}\left(f_{S}\right)+\ldots
\end{gathered}
$$

where $f_{0}=\tilde{\omega}_{0} / \omega_{c}\left(\tilde{\omega}_{0}=\omega_{0}-i v\right)$ and $f_{S}=\omega_{s} / \omega_{c}$ are respectively the normalized modulation and SPP frequencies $\left(F\left(f_{S}\right)=\tilde{F}\left(\omega_{s}\right)\right)$. We can illustrate the physical meaning of the field $E$ in the following limiting cases: $|y|<<1$ and $|y|>>1$.

Let us first consider the beam front. The time dependence of $y$ in $w(y)$ reveals a sharp maximum of the received wave field near the beam front when the SPP frequency $f_{S}$ approaches the modulation frequency $f_{0}(|y|<<1)$. At this time, the first term in $\mathrm{I}_{1}$, containing $w(y)(w(y) \approx 1$ for $|y|<<1)$, is dominant. One can see that the first term in $\mathrm{I}_{2}$ (with ratio $i / f_{S}$ ), which is identical to the wave field of a nonmodulated beam, is much smaller than $\mathrm{I}_{1}$ by a factor $\delta f / f$ which is small ( $\delta f$ represents the internal frequency half width of the received signal at frequency $f$ ). This justifies the fact that the efficiency of the wave field emission should be increased through the beam modulation (see first formula of (3)).

After the beam front pass, the emission at the modulation frequency will arrive to the receiver in a permanent regime mainly from a part of the beam which is connected to the receiver position through the corresponding group velocity vector. In the vicinity of the modulation frequency where $y$ is small, the signal of the modulated beam is greater than that of a nonmodulated one $(\chi=0)$. This increase of emission is produced from the beam part of length $L_{c}=8 u / \omega_{c} \delta f$. To reach the highest level of emitted signal, one should take care to keep in the beam a coherence of the beam electrons motion along this length $L_{c}$, so called "coherence length". The sharpness in time of the observable peak emission during the beam front pass increases proportionally to the square root $\sqrt{R}$ of the transverse distance from the beam to the receiver position; the value of the peak amplitude is inversely proportional to $\sqrt{R}$. Far from the beam front, the amplitude of the field at the modulation frequency will reach twice the value corresponding to the near beam front emission.

We supposed that $v<<\delta f$ : only in this case $|y|$ in $w(y)$ can be small. Otherwise, the effectively modulated region of the beam will be shorter than $L_{c}$, and will look like a single dot without internal structure; then the beam modulation will not produce an effective increase of the emitted wave field.

The essential assumption governing the amplitude of the signal in the previous calculations was the singular parallel velocity $u$ of the beam electrons. Now we will take into account the dependance of the phase of the signal, $\tilde{F}(\omega, u)$, on the parallel velocity (nonmonoenergetic beam). In expressions of fields as in (2) we must add an integration over the parallel velocity $u$. Thus let us find the condition for which the integration over the parallel velocity of the beam's electrons does not essentially diminish the signal level, i.e., when the SPP exists for some values of the parallel velocity of the beam particles. Applying the SPP method on $u$, one obtains the condition

$$
\left|v_{C \perp}\right| /\left|v_{G Z}\right|=R / Z
$$


In the case where roots common to (4) and $\partial \tilde{F}(\omega, u) / \partial \omega=0$ exist, one can predict that the field amplitude will not decrease crucially due to interference effects (which reduce the integrals over the rapidly oscillating functions to zero). One can see in (4) that the coherent emission can be received mostly from the near surrounding of the beam injection point (Fresnel zone of the order of $L_{c}$ ). Thus the level of emission depends crucially on the processes which occur inside the Fresnel zone around the gun. The size of this region as well as the frequency of the coherent signal depends on the beam pitch angle and on the resonance number $m$ at which the radiation takes place. In the case of a beam injection toward the receiver direction only emissions radiated at the normally shifted Doppler resonances $(m \geq 0)$ can be received on board the subsatellite as coherent high level signals in the ray approximation [9]. The wave packets radiated by a nonmonoenergetic beam at abnormally Doppler-shifted resonances $(m<0)$ (after the beam has passed through the parallel coordinate $Z$ of the receiver) do not satisfy the relation (4).

\section{EMISSION POWER [12-14]}

The electromagnetic field structure and the radiated power for whistler waves produced by a modulated beam interacting with the ionospheric plasma have been determined in the case of quasi-electrostatic whistlers of low frequency $\left(\omega<\omega_{c}<\omega_{p}, \quad k_{z}<<k_{\perp} \approx k\right)$ whose dispersion relation is

$$
\omega^{2}=\frac{\omega_{c}^{2}}{\left(1+\omega_{p}^{2} / k^{2} c^{2}\right)}\left(\frac{m_{e}}{m_{i}}+\frac{k_{z}^{2}}{k^{2}+\omega_{p}^{2} / c^{2}}\right)
$$

where $m_{e}$ and $m_{i}$ are respectively the electron and ion mass. The beam current with modulation at $\omega_{0}$ is taken as an external source in the Maxwell's equations which give after resolution the potentials in the Fourier space as a function of the beam current $\vec{j}_{b}\left(j_{b z, k} ; j_{b \perp, k}\right)$ and of the electromagnetic whistler dielectric constant $\varepsilon(\omega, k)$ through which two poles $k_{1}$ and $k_{2}$ will appear in the expressions of potentials

$$
\begin{gathered}
\varphi_{k}=\frac{4 \pi}{\varepsilon(\omega, k)}\left\{\frac{\left(k_{z} j_{b z, k} / \omega\right)}{\left(k^{2}+\omega_{p}^{2} / c^{2}\right)}-\frac{\omega_{p}^{2}}{c^{2} k^{4}} \frac{\left(\vec{\nabla} \times \vec{j}_{b \perp, k}\right) \cdot \vec{z}}{\omega_{c}}\right\} \\
A_{z k}=\frac{1}{\left(1+c^{2} k^{2} / \omega_{p}^{2}\right)} \frac{c k_{z}}{\omega} \frac{4 \pi}{\varepsilon(\omega, k)}\left\{\frac{\left(k_{z} j_{b z, k} / \omega\right)}{\left(k^{2}+\omega_{p}^{2} / c^{2}\right)}-\frac{\omega_{p}^{2}}{c^{2} k^{4}} \frac{\left(\vec{\nabla} \times \vec{j}_{b \perp, k}\right) \cdot \vec{z}}{\omega_{c}}\right\}+\frac{4 \pi c j_{b z, k}}{\omega_{p}^{2}\left(1+c^{2} k^{2} / \omega_{p}^{2}\right)}
\end{gathered}
$$

For sheared whistlers, only the axial z-component $A_{z k}$ of the potential vector is non negligeable. For the case of a parallel injection to the magnetic field, we consider a cylindric beam of finite radius $r_{b}$ whose current is along the magnetic field; in the case of oblique injection, the beam is modeled by an infinite-thin spiral structure of Larmor radius $R_{L}$. Through Fourier integration, we can obtain the analytical expressions of the potentials with Bessel and Hankel functions, and then derive the fields through $\vec{B}=\vec{\nabla} \times \vec{A}$ and $\vec{E}=-\vec{\nabla} \varphi-(1 / c) \partial \vec{A} / \partial t$. Asymptotic developments are performed in order to find simple analytical expressions for fields components near the beam and far from the beam. In the near beam approximation, in the case of parallel injection, the fields outside and inside the beam have been determined, for double or single poles of the Cherenkov resonance [12]. In the case of oblique injection, we have calculated the wave fields in the near external zone of the beam, for double and single poles in the Cherenkov and Doppler resonances [13]. Fields in the wave zone have also been derived [12-13].

A general expression of the power $P$ radiated by the modulated beam per unit length has been obtained in the case of sheared whistlers, in the region far from the Larmor radius, including both poles $k_{1}$ and $k_{2}$, the parallel group velocity, the Larmor radius, the resonance condition (through $k_{z}$ and the parallel beam velocity $u$ ) and the plasma parameters $\omega_{p}$ and $\omega_{c}$ 


$$
P=\frac{\Phi^{2}}{2 \pi} \frac{\omega_{p}^{2}}{\omega_{c}^{2}} \sum_{i=1}^{2} \frac{\left|v_{G Z i}\right| k_{i}^{5}}{\left(k_{1}^{2}-k_{2}^{2}\right)^{2}}\left[\left(\frac{k_{z} u}{\omega_{0}}\right)^{2}+\frac{1}{2}\left(\frac{R_{L} \omega_{p}}{c}\right)^{2}\left(\frac{\omega_{p}}{k_{i} c}\left(\frac{\omega_{p}^{2}}{k_{i}^{2} c^{2}}+1\right)\right)^{2}\right]\left[\frac{\omega_{p}^{2}}{k_{i}^{2} c^{2}}+1\right]
$$

where $\Phi$ is the typical whistler potential.

Figure 3 shows, for Cherenkov resonance and parallel injection, the variation of the radiated power as a function of the beam velocity $v_{b}$ : the maximum of power shows a sharp peak near the velocity corresponding to the double pole case $\left(v_{b} \approx 310^{7} \mathrm{~ms}^{-1}\right)$.

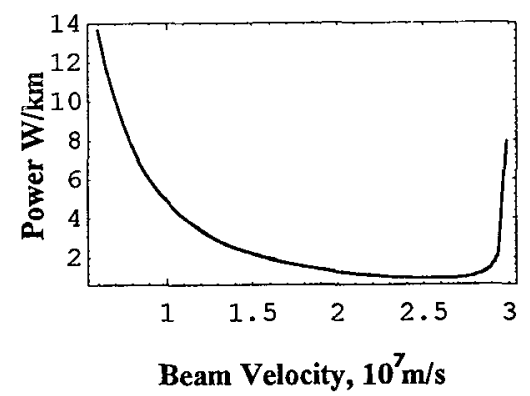

Figure 3: Power $P$ as a function of beam velocity $\mathrm{v}_{\mathrm{b}} ; \omega_{\mathrm{p}}=4.310^{6} \mathrm{~Hz}, \omega_{\mathrm{c}}=0.8610^{6} \mathrm{~Hz}, \omega_{\mathrm{o}} / 2 \pi=10^{4} \mathrm{~Hz}, \mathrm{I}_{\mathrm{b}}=0.1 \mathrm{~A}$.

\section{CONCLUSION}

The linear stage of the whistler wave excitation in the interaction between a modulated electron beam and a magnetized plasma has been investigated experimentally as well as theoretically. In the near future, we will study the saturation mechanisms and the nonlinear processes of the interaction.

\section{Acknowledgments}

B. Lundin and A. Volokitin acknowledge the financial support from DRET and CNRS during their visit to the Laboratoire de Physique des Gaz et des Plasmas of the University Paris South, Orsay, France.

\section{References}

[1] Kennel C.F. and Petschek H.E., J. Geophys. Res. 71 (1966) 1-28.

[2] Helliwell R.A., Adv. Space Res. 8(1) (1988) 279-284.

[3] Lavergnat J., The French-Soviet Experiment ARAKS: Main Results, Artificial Particle Beams in Space Plasma Studies (Plenum, New York, 1982) pp.87-90.

[4] Krafft C. et al., J. Phys. III France 1 (1991) 2047-2059.

[5] Krafft C., Matthieussent G. and Thévenet P., Euro. Lett. 24(9) (1993) 731-736.

[6] Krafft C. et al., Phys. Rev. Lett. 72 (1994) 649-652.

[7] Krafft C. et al., Phys. Plasmas 1(7) (1994) 2163-2171.

[8] Oraevsky V. et al., J. Atmos. Terr. Phys. 5(3) (1994) 423-431.

[9] Lundin B. et al., J. Geophys. Res. 99 (1994) 14987-15003.

[10] Lundin B., Krafft C. and Matthieussent G., J. Geophys. Res. 100 (1995) 3703-3713.

[11] Kraff C., Lundin B. and Matthieussent G., J. Phys. IV France, see this issue.

[12] Volokitin A., Krafft C. and Matthieussent G., Whistlers produced by a modulated electron beam: electromagnetic fields in the linear approach, to be published in Physics of Plasmas.

[13] Krafft C., Volokitin A. and Matthieussent G., Whistlers produced by a spiral modulated electron beam: linear approach, submitted to Physics of Plasmas.

[14 ] Volokitin A., Krafft C. and Matthieussent G., J. Phys. IV France, see this issue. 